\title{
Estratégias de coping e estresse ocupacional em profissionais de enfermagem: revisão integrativa
}

Coping strategies and occupational stress in nursing professionals: integrative review

Estrategias de coping y estrés ocupacional en profesionales de enfermería: revisión integrativa

Caroline Camargo Graça ${ }^{1}$, Ivete Palmira Sanson Zagonel $^{2}$

${ }^{1}$ Mestranda no Ensino nas Ciências da Saúde pela Faculdades Pequeno Príncipe- FPP Curitiba-Paraná

${ }^{2}$ Doutora em Enfermagem pela Universidade Federal de Santa Catarina. Docente do Programa de Mestrado em Ensino nas Ciências da Saúde da Faculdades Pequeno Príncipe - FPP. Curitiba-Paraná

\section{RESUMO}

O estudo teve como objetivo analisar as evidências científicas acerca do estresse ocupacional do profissional de enfermagem e estratégias de coping. Trata-se de uma revisão integrativa, realizada na base de dados online do Scientific Electronic Library Online (SCIELO). Foram utilizados como descritores em saúde: esgotamento profissional, estresse em enfermagem, coping. A partir dos critérios de seleção, foram obtidos 8 artigos para a análise dos resultados. A revisão indicou estratégias de enfrentamento (coping) como, ter condições mínimas de material, pessoal, estrutura e valorização, para se obter um trabalho adequado e assim não colocar em risco a vida do profissional. Detecção precoce do estresse profissional, sinais e sintomas que o

Autor de Correspondência:

*Ivete Palmira Sanson Zagonel .E-mail: ivete.zagonel@fpp.edu.br 
profissional pode apresentar, bem como, que o enfermeiro trabalha em condições estressantes diariamente. Conclui-se que há necessidade de assegurar bem-estar psicológico positivo, um ambiente de boas práticas e boas atitudes de segurança.

Palavras-chave: Estresse Ocupacional. Equipe de Enfermagem. Adaptação Psicológica.

\begin{abstract}
The study aimed to analyze the scientific evidence of occupational stress of nursing professionals and coping strategies. This is an integrative review, performed in the online database of the Scientific Electronic Library Online (SCIELO). The following health descriptors were used: professional exhaustion, nursing stress, coping. Based on the selection criteria, 8 articles were obtained for the analysis of the results. The review indicated coping strategies such as having minimum conditions of material, personnel, structure and valuation, to provide adequate service so as not to put the professional at risk. Early detection of occupational stress, signs and symptoms that the professional may present, as well as that the nurse works under stressful conditions daily. The conclusion is that it is necessary to ensure positive psychological wellbeing, an environment of good practice and good safety attitudes.
\end{abstract}

Keywords: Occupational Stress. Nursing, Team. Adaptation, Psychological. .

\title{
RESUMEN
}

El estudio tuvo como objetivo analizar la evidencia científica sobre el estrés laboral de los profesionales de enfermería y las estrategias de afrontamiento. Es una revisión integradora, realizada en la base de datos en línea de la Scientific Electronic Library Online (SCIELO). Se utilizaron los siguientes descriptores de salud: agotamiento profesional, estrés de enfermería, afrontamiento. A partir de los criterios de selección, se obtuvieron ocho artículos para el análisis de los resultados. La revisión indicó estrategias de afrontamiento, como tener condiciones mínimas de material, personal, estructura y valoración, para obtener un trabajo adecuado y, por lo tanto, no poner en peligro la vida del profesional. Detección temprana de estrés laboral, señales y síntomas que el profesional puede presentar, así como la enfermera que trabaja diariamente en condiciones estresantes. De ello, se deduce que es necesario garantizar un bienestar psicológico positivo, un entorno de buenas prácticas y buenas actitudes de seguridad.

Palabras clave: Estrés Laboral. Grupo de enfermería. Adaptación Psicológica. 


\section{INTRODUÇÃO}

O tema estresse tem sido discutido e difundido pelos diferentes meios de comunicação, como uma causa para acontecimentos que afligem a vida humana. Definido como qualquer estímulo que demande do ambiente externo ou interno e que exceda as fontes de adaptação de um indivíduo, ou sistema social. Há um aumento nas publicações de artigos e pesquisas científicas em relação à presença do estresse entre a equipe de enfermagem, uma vez que a abordagem do assunto está cada vez mais presente no cotidiano deste profissional. De acordo com dados da Organização Mundial da Saúde (OMS), aproximadamente 90\% da população mundial chega a ser afetada pelo estresse, o que faz com que esta problemática se torne em uma epidemia global ${ }^{1}$.

As profissões que exigem contato mais próximo com as pessoas, que por muitas vezes estão carregadas de envolvimento afetivo tais como medicina, psicologia, enfermagem e fisioterapia, estão mais sujeitas ao desenvolvimento do estresse no trabalho e da síndrome de Burnout. Devido à complexidade do curso de enfermagem e ao lidar com os limites humanos, o estudante desse curso frequentemente desenvolve sentimentos de incapacidade frente às atividades exigidas durante sua formação profissional $^{2}$.

O termo estresse ocupacional é provocado por elementos ligados ao trabalho, que compreende um conjuntodeatividades comvalores, intencionalidades, comportamentos e representações. Dessa forma, o trabalho proporciona crescimento, transformação, reconhecimento e independência pessoal, entretanto, as constantes mudanças impostas aos indivíduos podem desencadear também, problemas como insegurança, insatisfação, desinteresse e irritação ${ }^{3}$.

Evidencia-se que, ao deparar-se com um estressor, o organismo experimenta três fases: a primeira é a fase de alarme ou alerta, na qual o corpo identifica o estressor, e ativa o sistema neuroendócrino. A segunda fase, de adaptação ou resistência, é o período em que o organismo repara os danos causados pela reação de alarme e reduz os níveis hormonais. A terceira fase ocorre se o estressor permanecer presente, ou seja, é a fase de exaustão, que abrange o surgimento de uma doença associada ao estresse ${ }^{4}$.

Dessa forma, o estresse possui apresentação notória na atuação do enfermeiro, uma vez que a profissão lhe expõe a situações de tensão, devido às responsabilidades e complexidade das ações que lhe são exigidas. Desde o seu surgimento até os dias atuais, o trabalho da enfermagem está atrelado ao processo de dor, doença e morte, principalmente em instituições hospitalares, onde o contato diário com o paciente e a família é mais frequente. Assim, o profissional convive rotineiramente com a ansiedade, os sentimentos de perda e fragilidade dos pacientes, advindos de procedimentos dolorosos e invasivos, além de assistências desconfortáveis, em um ambiente considerado estranho e frio pelos pacientes. Todos estes fatores geram certa tensão psíquica, levando ao adoecimento do profissional ${ }^{1}$.

Um dos setores que possui fatores de estresse em maior intensidade são as unidades de urgência e emergência. Pode-se considerar que a maior fonte de satisfação no trabalho do enfermeiro nesses setores prende-se ao fato de que as suas intervenções auxiliam a manutenção da vida humana ${ }^{2}$.

Como principais estressores são citados os seguintes componentes: número reduzido de funcionários compondo a equipe de enfermagem; falta de respaldo institucional e profissional; sobrecarga de trabalho; necessidade de realização de atividades em tempo reduzido; indefinição do papel do profissional; descontentamento com o trabalho; falta de experiência por parte dos supervisores; falta de comunicação e compreensão por parte da supervisão de serviço; relacionamento com familiares; ambiente físico da unidade; tecnologia de equipamentos e assistência ao paciente em estado grave ${ }^{3}$. 
As organizações de saúde têm se sobressaído pela assistência a pacientes em situações cada vez mais críticas, que demandam respostas complexas a cada cuidado a ser prestado. Desse modo, o trabalho realizado em instituições de saúde requer competências clinicas especificas dos profissionais, que se deparam com transformações tecnológicas, mudanças no perfil epidemiológico, padrão demográfico, bem como exigências crescentes por parte dos pacientes ${ }^{5}$.

Diante das transformações organizacionais, somadas ao aumento significativo do estresse ocupacional, há exigência da equipe de enfermagem, em especial, do enfermeiro, de constante adequação para enfrentar as inovações e administrar a gravidade das situações. A identificação dos estressores no trabalho corresponde a um agente de mudança, uma vez que, desenvolvidas as possíveis estratégias de coping para minimizar seus efeitos, estas podem tornar o cotidiano do indivíduo mais produtivo, menos desgastante e, possivelmente, valorizá-lo mais como ser humano e como profissional ${ }^{1}$.

Os riscos à integridade física e, principalmente, à forma de gestão imposta por meio de metas de produtividade e organização do tempo, desconsideram os limites físicos e psicológicos dos trabalhadores ${ }^{6}$

Define-se coping como "a capacidade de enfrentamento e adaptação que permite ao ser humano reagir frente a comportamentos, pensamentos e emoções causados por eventos estressantes”. As respostas são características da reação do estresse e levam ao enfrentamento das demandas, por meio dos mecanismos de coping, que agem como direcionadores de respostas geradas pela avaliação e repercussão do evento na vida do indivíduo. O coping, quando utilizado corretamente, leva à redução, adaptação ou superação do problema. Em contrapartida, quando as estratégias utilizadas não são apropriadas para a situação, elas podem levar a um aumento do nível de estresse ${ }^{1}$.
Conviver com o estresse é um inevitável desafio. $\mathrm{O}$ enfermeiro em seu exercício profissional tem enfrentado dificuldades que comprometem o desempenho do seu trabalho e que também repercutem no seu lado pessoal. A indefinição do papel profissional pode ser relacionada como mais um dos elementos estressores ${ }^{7}$.

A resposta ao estresse precisa estar na mesma medida da ameaça e isto faz com que a pessoa mobilize energia suficiente para que o organismo se reequilibre. É necessário adotar estratégias relacionadas à diminuição das jornadas de trabalho e melhoria na infraestrutura das instituições ${ }^{8}$.

Se o indivíduo está capacitado a conviver efetivamente com o estresse, melhor será a aprendizagem. As pessoas necessitam de um nível mínimo de estresse para desenvolver suas atividades, já um alto nível de estresse é destrutivo, pois ocorre quando as estratégias de enfrentamento usuais são insuficientes para lidar com a situação ${ }^{1}$.

Nesta perspectiva, uma das consequências do estresse ocupacional é a Síndrome de Burnout ou Síndrome do Esgotamento Profissional. A enfermagem, devido à ocorrência dos inúmeros elementos estressores já expostos, trata-se de uma das profissões mais afetadas por esta síndrome ${ }^{9}$.

A justificativa em explorar esse tema flui do âmbito da importância de explorar as ocorrências do estresse profissional de enfermagem e as ocorrências do estresse profissional de enfermagem estratégias de coping no processo da manutenção da saúde do trabalhador de enfermagem, para melhor desenvolvimento do trabalho e, consequentemente, redução do nível de estresse, melhoria na qualidade de vida, considerando que o trabalho influencia diretamente no seu processo de bem-estar e/ou adoecimento.

Dado o exposto, a pesquisa objetivou analisar as evidências científicas disponíveis na literatura brasileira, acerca dos fatores que podem contribuir 
para a ocorrência do estresse profissional de enfermagem e as estratégias de coping.

\section{MÉTODO}

Para a obtenção dos resultados, optou-se pelo método de revisão integrativa. Consiste em uma metodologia específica de pesquisa em saúde que sintetiza um assunto ou referencial teórico para melhor compreensão e entendimento de um determinado assunto, permitindo uma ampla análise da literatura. Este método foi desenvolvido de acordo com os propósitos da Prática Baseada em Evidências (PBE), possuindo como pressuposto um rigoroso processo de síntese da realidade pesquisada ${ }^{10}$.

No geral, para a construção da revisão integrativa é preciso percorrer seis etapas distintas, as quais serão relacionadas a seguir.

A primeira etapa consiste na identificação do tema e seleção da hipótese ou questão de pesquisa para elaboração da revisão integrativa. Trata-se da escolha e definição do tema, objetivos e identificação das palavras-chave. O processo de elaboração da pesquisa integrativa se inicia com a definição de um problema e a definição de uma hipótese ou questão de pesquisa que apresente relevância para a saúde e enfermagem ${ }^{10}$. Este estudo foi construído, a partir da seguinte questão norteadora: "quais as evidências científicas disponíveis na literatura brasileira, acerca dos fatores que podem contribuir para a ocorrência do estresse profissional de enfermagem e as estratégias de coping?

A segunda etapa consiste no estabelecimento dos critérios de inclusão e exclusão de estudos/ amostragem ou busca na literatura. Esta etapa está intimamente atrelada à etapa anterior, uma vez que a abrangência do assunto a ser estudado determina o procedimento de amostragem, ou seja, quanto mais amplo for o objetivo da revisão, mais seletivo deverá ser o revisor para a inclusão da literatura a ser considerada ${ }^{10}$. Os dados foram coletados no mês de novembro de 2018, utilizando a seguinte combinação de descritores: esgotamento profissional, estresse em enfermagem, coping. As palavras-chave foram selecionadas no banco de Descritores em Ciências da Saúde (DeCS). Estabeleceram-se como critérios de inclusão para a realização desse estudo, artigos completos publicados no idioma português na base de dados online do Scientific Electronic Library Online (SCIELO), entre os anos de 2010 e 2018, e com a abordagem do tema proposto inicialmente. Como critérios de exclusão elencaram-se a falta do tema proposto, publicações em língua estrangeira, anterior ao ano de 2010 e apresentados na forma de resumos.

A terceira etapa refere-se à definição das informações a serem extraídas dos estudos selecionados/ categorização dos estudos. Esta etapa se caracteriza pela definição das informações retiradas de cada pesquisa, através de um instrumento para reunir e sintetizar as informações-chave ${ }^{10}$. Após a seleção das publicações iniciou-se um processo de leitura das mesmas, e em seguida foi efetuada a organização das informações. Inicialmente, obteve-se 23 artigos completos com a combinação das palavras-chave. Após a menção dos critérios para a seleção dos trabalhos, foi apresentada uma amostragem de 8 artigos para análise e discussão com a literatura.

A quarta etapa efetiva a avaliação dos estudos incluídos na revisão integrativa. Esta etapa é equivalente à análise dos dados, na qual há o emprego de ferramentas apropriadas para tal ação. Para garantir a fidedignidade da revisão, os estudos devem ser analisados detalhadamente e de forma criteriosa $^{10}$.

Para a extração das informações dos artigos científicos utilizou-se como instrumento um roteiro elaborado, validado ${ }^{11} \mathrm{e}$ mencionado ${ }^{12}$. Esta ferramenta foi adaptada para a presente pesquisa, considerando as seguintes características: dados de identificação do estudo, características metodológicas e principais 
evidências científicas apresentadas. Na sequência foi realizada a categorização dos dados através da leitura, a qual possibilitou evidenciar os principais tópicos (Quadro 1).

Quadro 1 - Categorização dos Artigos Selecionados

\begin{tabular}{|l|c|}
\hline \multicolumn{1}{|c|}{ Categorias } & Artigos Selecionados \\
\hline Perfil dos Profissionais de Enfermagem & Artigos 01, 02, 04, 05, 09, 13 \\
\hline $\begin{array}{l}\text { Formação e Tempo de Trabalho: características profissionais } \\
\text { que influenciam no estresse ocupacional }\end{array}$ & Artigos 01, 02, 03, 04, 05, 09, 13, 14 \\
\hline Estresse Ocupacional: sintomas físicos e psicossociais & Artigos 01, 02, 03, 04, 05, 09, 13, 15 \\
\hline
\end{tabular}

Fonte: a pesquisadora

$\mathrm{Na}$ quinta etapa efetivou-se a interpretação dos resultados. Corresponde à fase da discussão dos principais resultados a luz da literatura disponível sobre o tema, procurando explicações para cada conotação diferente e/ou conflitante ${ }^{10}$.

A sexta etapa corresponde à apresentação da revisão/ síntese do conhecimento. Nesta etapa, apresentase um apanhado das evidências disponíveis sobre a temática da pesquisa. A exposição deve ser clara e completa para permitir ao leitor avaliar criticamente os resultados encontrados ${ }^{10}$.

\section{RESULTADOS E DISCUSSÃO}

A finalidade do presente trabalho foi sintetizar a colocação de autores de artigos, previamente selecionados, que contemplam este assunto.

São descritos em quadros e tabelas os artigos selecionados, sendo destacados seu título, ano de publicação, local de publicação, autores e principais aspectos abordados (Quadro 2).
Entre os oito artigos que compõem esta amostra 3 (três) foram publicados na Revista da Escola de Enfermagem da Universidade de São Paulo (USP), representando $37,5 \%$ da amostra. No que se refere à abordagem metodológica dos estudos, 62,5\% corresponderam à abordagem quantitativa. Dois trabalhos apresentaram a abordagem qualitativa (25\%), e um com a abordagem quanti-qualitativa $(12,5 \%)$.

Quanto ao ano, percebe-se que os anos de 2011 e 2012 obtiveram a maior prevalência, somando $50 \%$ do total de estudos. Os anos de 2007, 2013, 2015, 2016 tiveram apenas uma publicação cada (12,5\%) (Gráfico 1).

Quanto à autoria dos artigos, todas as publicações $(100 \%)$ foram conduzidas por profissionais de enfermagem. Considerou-se a titulação do primeiro autor do artigo. Os pesquisadores com titulação de doutorado representaram $75 \%$ da amostra, seguidos pelos profissionais com especialização (12,5\%) e mestrado (12,5\%). Obteve-se sete dos primeiros autores dos artigos, que atuam em Instituições de Ensino Superior e um em hospital. 
Quadro 2 - Caracterização dos Artigos Selecionados

\begin{tabular}{|c|c|c|c|}
\hline Periódico/ano & Autores & Título & Objetivos \\
\hline $\begin{array}{l}\text { Revista Escola } \\
\text { Enfermagem Anna } \\
\text { Nery } \\
2007\end{array}$ & $\begin{array}{c}\text { Spindola T; } \\
\text { Martins ERC. }\end{array}$ & $\begin{array}{l}\text { O estresse e a enfermagem } \\
\text { - a percepção das } \\
\text { auxiliares de enfermagem } \\
\text { de uma instituição }\end{array}$ & $\begin{array}{l}\text { Identificar como as auxiliares de } \\
\text { enfermagem relacionam o estresse } \\
\text { às suas atividades diárias. }\end{array}$ \\
\hline $\begin{array}{l}\text { Revista Escola de } \\
\text { Enfermagem USP } \\
2011\end{array}$ & $\begin{array}{l}\text { Guido LA; } \\
\text { Linch GFC; } \\
\text { Pitthan LO; } \\
\text { Umann J. }\end{array}$ & $\begin{array}{l}\text { Estresse, coping e estado } \\
\text { de saúde entre enfermeiros } \\
\text { hospitalares }\end{array}$ & $\begin{array}{l}\text { Identificar estressores, nível } \\
\text { de estresse dos enfermeiros, } \\
\text { estado geral de saúde e formas de } \\
\text { enfrentamento utilizados pelos } \\
\text { enfermeiros no âmbito de trabalho. }\end{array}$ \\
\hline $\begin{array}{l}\text { Revista da Escola } \\
\text { de Enfermagem da } \\
\text { USP } \\
2011\end{array}$ & $\begin{array}{l}\text { Farias SMC; } \\
\text { Teixeira OLC; } \\
\text { Moreira W; } \\
\text { Oliveira MAF; } \\
\text { Pereira MO. }\end{array}$ & $\begin{array}{l}\text { Caracterização dos } \\
\text { sintomas físicos de } \\
\text { estresse na equipe de } \\
\text { pronto atendimento }\end{array}$ & $\begin{array}{l}\text { Identificar os fatores } \\
\text { desencadeantes de estresse na } \\
\text { equipe de enfermagem que trabalha } \\
\text { na unidade de pronto atendimento } \\
\text { adulto e infantil de um hospital. }\end{array}$ \\
\hline $\begin{array}{l}\text { Revista Gaúcha de } \\
\text { Enfermagem } \\
2012\end{array}$ & $\begin{array}{l}\text { Seleghim MR; } \\
\text { Mombelli MA; } \\
\text { Oliveira MLF; } \\
\text { Waidman MAP; } \\
\text { Marcon SS. }\end{array}$ & $\begin{array}{l}\text { Sintomas de estresse } \\
\text { em trabalhadoras de } \\
\text { enfermagem de uma } \\
\text { unidade de pronto- socorro }\end{array}$ & $\begin{array}{l}\text { Identificar a associação de dados } \\
\text { sociodemográficos, ocupacionais } \\
\text { e econômicos com a presença } \\
\text { de sintomas de estresse em } \\
\text { trabalhadores de enfermagem de } \\
\text { uma unidade de pronto-socorro. }\end{array}$ \\
\hline $\begin{array}{l}\text { Acta Paulista de } \\
\text { Enfermagem } \\
2012\end{array}$ & $\begin{array}{l}\text { França SPS; } \\
\text { Martino MMF; } \\
\text { Aniceto EVS; } \\
\text { Silva LL. }\end{array}$ & $\begin{array}{l}\text { Preditores da Síndrome de } \\
\text { Burnout em enfermeiros de } \\
\text { serviços de urgência pré- } \\
\text { hospitalar }\end{array}$ & $\begin{array}{l}\text { Analisar os preditores da Síndrome } \\
\text { de Burnout apresentados por } \\
\text { enfermeiros dos serviços de urgência } \\
\text { pré-hospitalar móvel. }\end{array}$ \\
\hline $\begin{array}{l}\text { Revista da Escola } \\
\text { de Enfermagem da } \\
\text { USP } \\
2013\end{array}$ & $\begin{array}{l}\text { Oliveira JDS; } \\
\text { Achieri JC; } \\
\text { Pessoa-Júnior JM; } \\
\text { Miranda FAN; } \\
\text { Almeida MG. }\end{array}$ & $\begin{array}{l}\text { Representações sociais } \\
\text { de enfermeiros acerca do } \\
\text { estresse laboral em um } \\
\text { serviço de urgência }\end{array}$ & $\begin{array}{l}\text { Apreender as representações dos } \\
\text { enfermeiros sobre o seu trabalho em } \\
\text { serviço de urgência e a relação com } \\
\text { o estresse. }\end{array}$ \\
\hline $\begin{array}{l}\text { Revista Brasileira } \\
\text { de Terapia } \\
\text { Intensiva } \\
2015\end{array}$ & $\begin{array}{l}\text { Silva JLL; } \\
\text { Soares RS; } \\
\text { Costa FS; } \\
\text { Ramos DS; } \\
\text { Lima FB; } \\
\text { Teixeira LR. }\end{array}$ & $\begin{array}{l}\text { Fatores psicossociais } \\
\text { e prevalência da } \\
\text { síndrome de Burnout } \\
\text { entre trabalhadores de } \\
\text { enfermagem intensivistas }\end{array}$ & $\begin{array}{l}\text { Analisar a prevalência de Síndrome } \\
\text { de Burnout entre trabalhadores de } \\
\text { enfermagem intensivistas, traçando } \\
\text { relações com fatores psicossociais e } \\
\text { sociodemográficos. }\end{array}$ \\
\hline $\begin{array}{l}\text { Revista Texto } \\
\text { e Contexto de } \\
\text { Enfermagem } \\
2016\end{array}$ & $\begin{array}{l}\text { Bublitz S; } \\
\text { Guido LA; } \\
\text { Lopes LFD; } \\
\text { Freitas EO. }\end{array}$ & $\begin{array}{l}\text { Associação entre } \\
\text { estresse e características } \\
\text { sociodemográficas e } \\
\text { acadêmicas de estudantes } \\
\text { de enfermagem }\end{array}$ & $\begin{array}{l}\text { Verificar a associação entre estresse } \\
\text { de discentes de enfermagem e as } \\
\text { características sociodemográficas e } \\
\text { acadêmicas dos mesmos. }\end{array}$ \\
\hline
\end{tabular}


Gráfico 1 - Distribuição dos artigos selecionados, quanto ao ano de publicação.

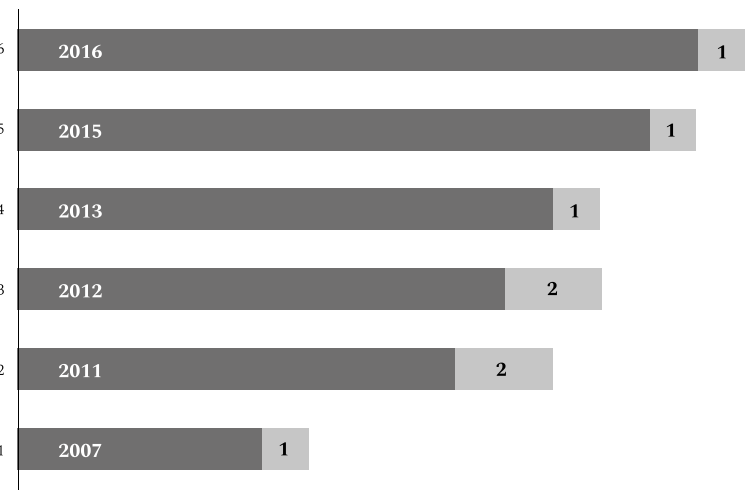

Fonte: a pesquisadora

Considerando a análise de sexo, um dos artigos não apresentou este perfil. Estudos indicaram a homogeneidade entre os participantes do estudo, ou seja, $50 \%$ de cada sexo ${ }^{12,13}$. Houve predominância do sexo feminino, frente ao sexo masculino entre os participantes do estudo ${ }^{5,15}$. Outro estudo observou maior prevalência de mulheres exercendo a profissão de enfermagem, indicando que as mulheres, além das atividades profissionais na dinâmica das organizações, aliam suas funções aos papéis de esposas e mães, inter-relacionando as atividades laborais com os familiares ${ }^{16}$. Considerando que a maioria dos profissionais de enfermagem é mulher e a condição feminina, por sua vez, ainda agrega outras atividades no lar, ocorre um sinergismo dentre as atribuições desta profissional, que pode propiciar o estresse $^{22}$.

No que diz respeito à idade dos participantes, a partir da análise dos artigos selecionados observa-se a média de idade entre 30 e 40 anos dos profissionais entrevistados ${ }^{5,13,14,15}$. Entretanto, nota-se a inserção de profissionais cada vez mais jovens no mercado de trabalho, o qual encontra-se mais competitivo e exige maior grau de especialização e conhecimento. Tais exigências podem exceder as capacidades de adaptações desta população específica, muitas vezes, sem experiência prévia com o ambiente laboral, levando-os a alto nível de estresse decorrente das exigências do setor de trabalho ${ }^{14,17}$.

Em todos os artigos apresentados os autores trazem a participação dos enfermeiros de maneira significativa na equipe de saúde $e^{5,12,13,15,18}$. O fator escolaridade com ensino superior completo pode ser considerado um fator determinante para a ocorrência do estresse ocupacional pelas atribuições como enfermeiro ${ }^{15}$. O enfermeiro constantemente em suas atividades laborais é responsável por funções "que envolvem outras profissões, como o gerenciamento do setor e da equipe, a educação em saúde da equipe, do paciente/cliente e da família, além da assistência direta ao paciente" ${ }^{19}$.

Aliado a esta condição, em se tratando de unidades de urgência e emergência é possível acrescentar à sobrecarga de trabalho do enfermeiro, rotinas aceleradas, jornadas noturnas, tomada de decisões rápidas, estrutura física com luzes artificiais, os ambientes fechados de trabalho, alarmes sonoros contínuos e intermitentes, entre outros fatores ${ }^{20}$.

De forma paralela, o tempo de trabalho no setor, bem como a existência de vínculos empregatícios concomitantes, também parecem interferir de forma negativa no surgimento do estresse ocupacional ${ }^{5,12,13,15}$. Verificou-se que $53,9 \%$ dos trabalhadores de enfermagem possuem dupla jornada de trabalho, o que leva ao acúmulo de atividades profissionais ${ }^{21}$. A dupla jornada de trabalho, muitas vezes, leva por si só à sobrecarga de trabalho. Desta forma, a exigência em excesso, fonte geradora de estresse, leva a diminuição do rendimento da trabalhadora e do tempo dispensado para seu autocuidado e lazer. Formando-se, assim, uma situação em que o trabalho gera o estresse e a diminuição do autocuidado pode gerar estresse crônico ${ }^{22}$.

São inúmeros os sintomas físicos e psicossociais advindos do estresse ocupacional em trabalhadores da área de enfermagem. O esgotamento profissional 
e a baixa realização pessoal tornam o enfermeiro um profissional "mecânico" e sem perspectivas positivas para o seu futuro profissional ${ }^{14}$. Dentre os sinais e sintomas mais frequentes destacam-se a queda em sua produtividade, levando a alterações cardiovasculares, fadiga, enxaqueca, insônia, dores musculares, úlcera péptica, ansiedade, irritabilidade, depressão, além de interferir na relação com a família.

A presença de sensibilidade emotiva excessiva, dúvida quanto a si próprio, diminuição da libido, além de mal-estar generalizado, formigamento das extremidades, desgaste físico, mudança no apetite, problemas dermatológicos, tontura, problemas de memória e cansaço frequentes ${ }^{15}$.

Em outro estudo, além de serem apontados alguns dos sintomas citados anteriormente, houve a apresentação de desânimo pela manhã, insônia e dificuldade para manter o sono, dores gástricas, tremores musculares, fôlego curto, dispneia, taquicardia, sudorese, rubor facial, sendo que os mais citados foram as cefaleias frequentes, tensões musculares e a fadiga ${ }^{5}$.

Estudos realizados referem os fatores predisponentes que levam ao desenvolvimento do estresse ocupacional, tais como a desmotivação do profissional, a falta de reconhecimento do enfermeiro e a baixa remuneração salarial. Tais condições colaboram para o aparecimento das manifestações clínicas e interferem negativamente no desempenho de suas atividades laborais ${ }^{13,18}$.

Em estudo com 474 enfermeiros observou-se que o impacto do bem-estar psicológico nas atitudes de segurança foi mediado significativamente pela qualidade do ambiente de prática. $\mathrm{O}$ uso de estratégias de enfrentamento orientadas à abordagem foi significativamente preditivo de bemestar psicológico positivo, um ambiente de boas práticas e boas atitudes de segurança ${ }^{23}$.

Sendo assim, analisando os dados encontrados nos estudos selecionados nota-se que há vários sinais e sintomas que mostram o estresse no profissional, o que interfere negativamente no trabalho, no convívio pessoal e profissional, tornando o enfermeiro um profissional frio e mal adaptado ao trabalho. De maneira geral, percebe-se que o excesso de trabalho pode produzir gradualmente a exaustão emocional e física, reduzindo sua energia no que diz respeito à eficiência, saúde e bem-estar.

\section{CONCLUSÕES}

Com o presente estudo é possível concluir que a enfermagem trabalha em condições estressantes, e que para dedicar-se a uma prestação de assistência efetiva e eficaz, fazem-se necessárias condições mínimas de material, pessoal, estrutura e valorização.

Ainda pode-se observar que a idade pode interferir no serviço, pois quanto mais novo for o profissional maior é sua insegurança consigo mesmo, que poderá torná-lo um candidato exposto aos agentes estressores com que convive diariamente. Mas, por outro lado, o tempo de serviço também torna-se um grande agente que pode levar o indivíduo ao estresse, pois a rotina, a convivência todos os dias com fatores estressantes, com cobranças rigorosas, pressões por parte tanto dos pacientes, dos acompanhantes, como também dos colegas de profissão podem levar o indivíduo a se sentir desmotivado e interferir negativamente no desempenho de suas atividades profissionais.

Então, conclui-se que o surgimento do estresse em profissionais da área de enfermagem, geralmente está associado a um conjunto de fatores, e não somente a uma predisposição simples. Tais condições podem ser caracterizadas como aspectos sociais, pessoais, condições precárias de trabalho, falta de valorização, faixa etária, período de trabalho, carga horária diária, entre outros. Essa exposição ocupacional acarreta o adoecimento do profissional em âmbito psicológico, emocional e social, tornando-o uma pessoa fria, sem 
paciência e sem vontade de trabalhar.

Sendo assim, essa pesquisa mostra que há a necessidade de implementar estratégias de coping, a fim de ajudar no enfrentamento do estresse vivenciado pelo profissional de enfermagem, diminuindo as fontes de estresse e melhorando a qualidade de vida do profissional responsável pelo cuidado.

\section{REFERÊNCIAS}

Guido LA, Linch GFC, Pitthan LO, Umann J. Estresse, coping e estado de saúde entre enfermeiros hospitalares. Ver. Escola Enferm. USP. 2011; 45(6):1434-9.

Almeida AMO, Lima AKG, Vasconcelos MGF, Lima ACS, Oliveira GYM. Estresse ocupacional em enfermeiros que atuam em cuidados ao paciente crítico. Rev. Enferm. UFPE. [online]. 2016: 10(5), 1663-1671.

Batista KM, Bianchi ERF. Estresse do enfermeiro em unidade de emergência. Rev Latino-am Enferm. 2006; 14(4):534-9.

Panizzon C, Luz AMH, Fensterseifer LM. Estresse da equipe de enfermagem de emergência clínica. Rev Gaúcha Enferm. 2008; 29(3), 391-98.

Farias SMC, Teixeira OLC, Moreira W, Oliveira MAF, Pereira MO. Caracterização dos sintomas físicos de estresse na equipe de pronto atendimento. Rev Escola Enferm USP. 2011; 45(3), 722-729.

Melo CF, Cavalcante AKS, Façanha KQ. Invisibilização do adoecimento psíquico do trabalhador: limites da integralidade na rede de atenção à saúde. Trab. Educ. Saúde, 2019; 17(2): e0020132.

Stacciarini JM, Tróccoli BT. O estresse na atividade ocupacional do enfermeiro. Rev Latino-am Enferm. 2001; 9(2): 17-25.

Andrade FM, Oliveira LB, Corrêa MCD, Santos CB, Silva JO, Maciel LFA, Rocha RJC, Monteiro ÍA, Magalhães DOL, Ferreira TA, Tolentino RM. Síndrome de Burnout em profissionais de enfermagem no ambiente hospitalar: uma revisão integrativa. REAS [Internet]. Available from: https://www.acervomais.com.br/index.php/saude/article/ view/334

Bezerra FN, Silva TM, Ramos VP. Occupational stress of nurses in emergency care: an integrative review of the literature. Acta Paul. enferm. 2012; 25(spe2): 151-156. ISSN 0103-2100

Mendes KDS, Silveira RCCP, Galvão CM. Revisão integrativa: método de pesquisa para a incorporação de evidências na saúde e na enfermagem. Texto \& Contexto Enferm. 2008; 17(4): 758-764.

Ursi ES, Galvão CM. Prevenção de lesões de pele no perioperatório: revisão integrativa da literatura. Rev Latino-am Enferm. 2006; 14(1):124-31.

Pedersoli CE, Dalri CB, Silveira RCPDC. O uso da máscara laríngea pelo enfermeiro na ressuscitação cardiopulmonar: revisão integrativa da literatura [dissertação]. Ribeirão Preto (SP): Escola de Enfermagem de Ribeirão Preto da Universidade de São Paulo. Programa de Pós-Graduação em Enfermagem Fundamental, 2009.

Oliveira JDS, Alchieri JC, Pessoa Júnior J.M, Miranda FAN, Almeida MG. Representações sociais de enfermeiros acerca do estresse laboral em um serviço de urgência. Rev Escola Enferm USP. 2013; 47(4): 984-9.

Silva JLL, Soares RS, Costa FS, Ramos DS, Lima FB, Teixeira LR. Fatores psicossociais e prevalência da síndrome de Burnout entre trabalhadores de enfermagem intensivistas. Rev Bras de Ter Intensiva. 2015; 27(2): 125133.

Seleghim MR, Mombelli MA, Oliveira MLF, Waidman MAP, Marcon SS. Sintomas de estresse em trabalhadoras de enfermagem de uma unidade de pronto socorro. Rev Gaúcha Enferm. 2012; 33(3): 165-173.

Spindola T, Martins ERC. O estresse e a enfermagem - a percepção das auxiliares de enfermagem de uma instituição pública. Esc Anna Nery R Enferm. 2007; 11(2): 212-219.

Bublitz S, Guido LA, Lopes LFD, Freitas EO. Associação entre estresse e características sociodemográficas e acadêmicas de estudantes de enfermagem. Texto Contexto Enferm. 2016; 25(4): e2440015

França SPS, Martino MMF, Aniceto EVS, Silva LL. Preditores da síndrome de Burnout em enfermeiros de serviços de urgência pré-hospitalar. Acta Paul 
Enferm. 2012; 25(1): 68-73.

Pereira DS, Araújo TSSL, Gois CFL, Gois Júnior JP, Rodriguez EOL, Santos V. Estressores laborais entre enfermeiros que trabalham em unidades de urgência e emergência. Rev Gaúcha Enferm. 2013; 35(1): 55-61.

Versa GLGS, Murassaki ACY, Inoue KC, Melo WA, Faller JW, Matsuda LM. Estresse ocupacional: avaliação de enfermeiros intensivistas que atuam no período noturno. Rev Gaúcha Enferm. 2012; 33(2): 78-85.

Araújo TM, Aquino E, Menezes G, Santos CO, Aguiar L. Aspectos psicossociais do trabalho e distúrbios psíquicos entre trabalhadoras de enfermagem. Rev Saúde Pública. 2003; 37(4): 424-433.

Montanholi LL, Tavares DMS, Oliveira GR. Estresse: fatores de risco no trabalho do enfermeiro hospitalar. Rev Bras Enferm. 2006; 59(5): 661-5.

Lee TSH, Tzeng WC, Chiang HH. Impact of coping strategies on nurses' well-being and practice. Journal of Nursing Scholarship. 2019; 51(2): 195-204. 